\title{
Proliferation and invasion of ovarian cancer cells are suppressed by knockdown of TRIM11
}

\author{
YAN CHEN $^{1}$, JIN SUN $^{1}$ and JUNYAN MA ${ }^{2}$ \\ ${ }^{1}$ Clinical Laboratory and ${ }^{2}$ Women's Reproductive Health Laboratory of Zhejiang, The Women's Hospital, \\ Zhejiang University School of Medicine, Hangzhou, Zhejiang 310006, P.R. China
}

Received March 7, 2017; Accepted May 29, 2017

DOI: $10.3892 / \mathrm{ol} .2017 .6432$

\begin{abstract}
Tripartite motif-containing (TRIM)11, an E3 ubiquitin ligase, is involved in the development of the nervous system. As an oncogene, it has also been identified in glioma, lung and colon cancer. However, few studies have been conducted on TRIM11 expression and functions in ovarian cancer. In the present study, we found that TRIM11 expression was obviously elevated in ovarian cancer tissues compared to adjacent non-cancerous tissues. Depletion of TRIM11 in A2780 and SK-OV-3 ovarian cancer cells by transfection of specific small interfering RNA significantly suppressed proliferation and inhibited invasion of cells, even induced apoptosis as indicated by both Cell Counting Kit-8, Annexin V/propidium iodide staining and Transwell assay. Furthermore, we explored the underlying mechanisms. Knockdown of TRIM11 not only affects the expression of cell apoptosisrelated (Bcl-2 and Bax) and invasion-related proteins [matrix metalloproteinase (MMP)-2 and MMP-9], but also reduced the phosphorylation levels of ERK and AKT. In conclusion, we showed that TRIM11 was upregulated in ovarian cancer tissue samples and that TRIM11 may serve as an oncogene in ovarian cancer.
\end{abstract}

\section{Introduction}

Ovarian cancer is regarded as the most lethal gynecologic malignancy and a serious threat to women's health worldwide (1). The treatment for ovarian cancer patients at an early stage (stages I or II) is effective, with 5-year survival of up to $90 \%$. However, cases diagnosed at an end-stage are approximately $70 \%$ (stages III or IV) with survive for 5 years of $\leq 30 \%$ (2). The lacks of effective screening strategies for early

Correspondence to: Dr Junyan Ma, Women's Reproductive Health Laboratory of Zhejiang, The Women's Hospital, Zhejiang University School of Medicine, 1 Xueshi Road, Hangzhou, Zhejiang 310006, P.R. China

E-mail: majunyan81@163.com

Key words: tripartite motif-containing 11, ovarian cancer, PI3K/AKT, migration, invasion detection contribute to the delay in diagnosis and the high mortality rate of ovarian carcinogenesis. Thus, deeper research and better understanding of the mechanisms of ovarian cancer will facilitate the identification of reliable diagnostic markers and the development of effective treatment.

Tripartite motif-containing (TRIM) 11 is a kind of TRIM protein. TRIM proteins have a tripartite motif consisting of gRING-finger, B-Box and coiled-coil domains (3). TRIM11 is identified as an E3 ubiquitin ligase in humans (4), PHOX2B (paired-like homeobox 2b) (5) and PAX6 (paired-box gene 6) (6), thus participate in the development of the nervous system. Moreover, TRIM11 interacts with and destabilizes ARC105, which suppresses ARC105-mediated transcriptional activation (7). Recently, it has been reported that TRIM11 is upregulated and play an oncogenic function in glioma (8), lung cancer (9) and colon cancer (10). Several members of TRIM proteins, including TRIM25 (11), TRIM27 (12) and TRIM29 (13), were elevated in ovarian cancer, while TRIM16 expression (14) decreased in ovarian cancer. However, the expression, and functions of TRIM11 in ovarian cancer have been insufficiently characterized.

The present study concluded that the level of TRIM11 expression is higher in ovarian cancer tissues than in matched adjacent non-cancerous tissues. We then investigated the biological functions of TRIM11 by knocking down its expression in ovarian cancer cell lines. The TRIM11 knockdown significantly suppressed proliferation and inhibited invasion of cells, and induced apoptosis. Additionally, depletion of TRIM11 inhibited the activities of AKT and ERK. TRIM11 is a potent oncogene in ovarian cancer.

\section{Patients and methods}

Specimen collection and cell culture. A total of 40 patients (median age, 53 years; range, 30-68 years) with ovarian cancer who underwent surgical resection at Clinical Laboratory, the Women's Hospital, Zhejiang University School of Medicine were registered in this project. Ovarian cancer tissues and matched non-cancerous tissues were obtained from the above 40 patients after written informed consent was obtained. Tissue samples were stored in liquid nitrogen at $-80^{\circ} \mathrm{C}$ before used. This study was reviewed and supported by the Ethics Committee of the Women's Hospital, Zhejiang University School of Medicine. 
The human ovarian A2780 and SK-OV-3 cell lines were provided by the Cell Bank of the Shanghai Institute for Biological Sciences, Chinese Academy of Sciences (Shanghai, China) and cultured in Dulbecco's modified Eagle's medium (DMEM) supplemented with $10 \%$ fetal bovine serum (FBS) (both from Gibco, Los Angeles, CA, USA) and $1 \%$ penicillin/streptomycin $2 \mathrm{mM}$ L-glutamine. Both cell lines were maintained at $37^{\circ} \mathrm{C}$ in a $5 \% \mathrm{CO}_{2}$ incubator.

RNA extraction and real-time PCR. Total RNA was isolated from tissue samples or cell lines using TRIzol (Invitrogen, Carlsbad, CA, USA) per the manufacturer's instructions. After removing the residual DNA with DNase I (Roche Diagnostics, Indianapolis, IN, USA), cDNA was synthesized by reverse transcription of $2 \mu \mathrm{g}$ of total RNA by using M-MLV Reverse Transcriptase kit (Thermo Fisher Scientific, Inc., Rockford, IL, USA). Real-time PCR was conducted in triplicate on an ABI 7300 machine (Applied Biosystems, Foster City, CA, USA) with SYBR ${ }^{\circledR}$ Green PCR Master mix (Thermo Fisher Scientific, Inc.). GAPDH served as an internal control. The sequences of PCR primers are as follows: TRIM11, 5'-CACC TAAGCTGCACAGTTCC-3' and 5'-GGCTGCCTCCTAAT TCTTCC-3'; GAPDH, 5'-CACCCACTCCTCCACCTTTG-3' and 5'-CCACCACCCTGTTGCTGTAG-3'.

Western blot analysis. Using RIPA lysis buffer, protein can be extracted from cell lines $(20 \mathrm{mM}$ Tris- $\mathrm{HCl}, \mathrm{pH} 7.4,150 \mathrm{mM}$ $\mathrm{NaCl}, 1 \mathrm{mM}$ EDTA and $0.5 \%$ Nonidet P-40) with fresh-added protease inhibitor cocktail (Roche Diagnostics). Protein concentrations depend on Bradford assay. Then equal number of proteins was separated for each sample by SDS-PAGE and blotted onto nitrocellulose filter membranes (EMD Millipore, Bedford, MA, USA). The membranes were incubated in 5\% skim milk for $30 \mathrm{~min}$, at room temperature, after that, with primary antibodies at $4^{\circ} \mathrm{C}$ overnight. Rabbit polyclonal TRIM11 antibody (dilution, 1:500; cat. no. ab111694), rabbit polyclonal MMP-9 antibody (dilution, 1:500; cat. no. ab73734); rabbit polyclonal MMP-2 antibody (dilution, 1:500; cat. no. ab37150); rabbit monoclonal Bcl-2 antibody (dilution, 1:500; cat. no. ab32124); rabbit monoclonal Bax antibody (dilution, 1:500; cat. no. ab32503); rabbit polyclonal Akt antibody (dilution, 1:500; cat. no. ab8805) and rabbit monoclonal pAkt antibody (dilution, 1:500; cat. no. ab81283); rabbit polyclonal ERK antibody (dilution, 1:500; cat. no. ab196883) and rabbit polyclonal p-ERK antibody (dilution, 1:500; cat. no. ab192591) and rabbit polyclonal GAPDH antiboody (dilution, 1:500; cat. no. ab37168) were all purchased from Abcam (Cambridge, MA, USA). Subsequently, the membranes were applied with secondary goat anti-rabbit (HRP) IgG antibody (dilution, 1:2,000; cat. no. ab6721; Abcam) and detected with the enhanced chemiluminescence system (Bio-Rad, Richmond, CA, USA). GAPDH was used as loading control. Band intensities were assessed on ImageJ software version 1.6 (http://rsb.info.nih.gov/ij/; National Institutes of Health, Bethesda, MD, USA).

RNA interference. TRIM11 small interfering RNA (siRNA) (siTRIM11, 5'-CUAUUCAUCUUUCCCGAGA-3') and negative control siRNA (siNC) were from GenePharma (Shanghai, China). A2780 and SK-OV-3 cells were transfected with siRNA or siNC by using Lipofectamine 2000 (Invitrogen) following the manufacturer's instructions. At $48 \mathrm{~h}$ post-transfection, by real-time PCR and western blot analysis, the knockdown efficiency was assessed.

Cell proliferation assay. The evaluation of cell proliferation was made by using Cell Counting Kit- 8 (CCK-8; Beyotime, Haimen, China) following the manufacturer's instructions. A2780 and SK-OV-3 cells were seeded onto 96-well plates (2,000 cells/well) and transfected with siTRIM11 or siNC. After incubation for 0, 24, 48 and $72 \mathrm{~h}, \mathrm{CCK}-8$ reagent was added and incubated at $37^{\circ} \mathrm{C}$ for $1 \mathrm{~h}$. At $450 \mathrm{~nm}$, the absorbance was measured on a microplate reader (Perlong, Beijing, China) with a reference wave length at $655 \mathrm{~nm}$.

Cell apoptosis analysis. The proportion of apoptotic cells were determined by using Annexin V-fluorescein isothiocyanate (FITC)/propidium iodide (PI) apoptosis kit (Nanjing KeyGen Biotech Co., Ltd., Nanjing, China) following the manufacturer's instructions. Cells cultured in 6-well plates were transfected with siTRIM11 or siNC. After incubating for $48 \mathrm{~h}$, cells were harvested, labeled with Annexin V-FITC and PI, and analyzed using a FACScan flow cytometry (BD Biosciences, San Jose, CA, USA) within $1 \mathrm{~h}$.

Transwell invasion assays. The Transwell assay of cell invasion was with $8-\mu \mathrm{m}$-pore filters pre-coated with Matrigel (Corning, New York, NY, USA). Cells were treated with siTRIM11 or siNC. At $24 \mathrm{~h}$ after treatment, cells were collected and plated in the upper chamber $\left(5 \times 10^{4}\right.$ cells/well $)$ with DMEM medium. The lower chamber contained DMEM supplemented with 10\% FBS. At $22 \mathrm{~h}$, the non-migrating cells were removed, and the filters fixed in $10 \%$ formalin, stained by $0.5 \%$ crystal violet. Using an inverted microscope, cells in 5-random fields were counted.

Statistical analysis. Statistical analysis were carried out by GraphPad Prism 6 (GraphPad Software, San Diego, CA, USA). All values are presented as the mean \pm standard deviation. One-way analysis of variance was used to test the statistical differences. A value of $\mathrm{P}<0.05$ was considered to indicate a statistically significant difference.

\section{Results}

Upregulation of TRIM11 in ovarian cancer tissues. Real-time PCR analysis was performed in 40 pairs of ovarian cancer and adjacent non-tumorous tissues and the $\log 2$ tumor/normal ratio of each cancer specimen was calculated. As shown in Fig. 1, overexpression of TRIM11 was found in $77.5 \%$ (31/40) of the tested ovarian cancer tissues.

Knockdown of TRIM11 expression by siRNA transfection. To investigate the biological functions of TRIM11 in ovarian cancer progression, siRNA transfection was used to knock down TRIM11 expression in A2780 and SK-OV-3 cells. As shown in Fig. 2A, siNC did not change the mRNA expression of TRIM11 compared to wild-type cells. TRIM11 siRNA (siTRIM11) significantly reduced TRIM11 mRNA expression compared to cells transfected with siNC in both cell lines. Western blot analysis revealed that siTRIM11 efficiently inhibited the protein expression of TRIM11 (Fig. 2B). 


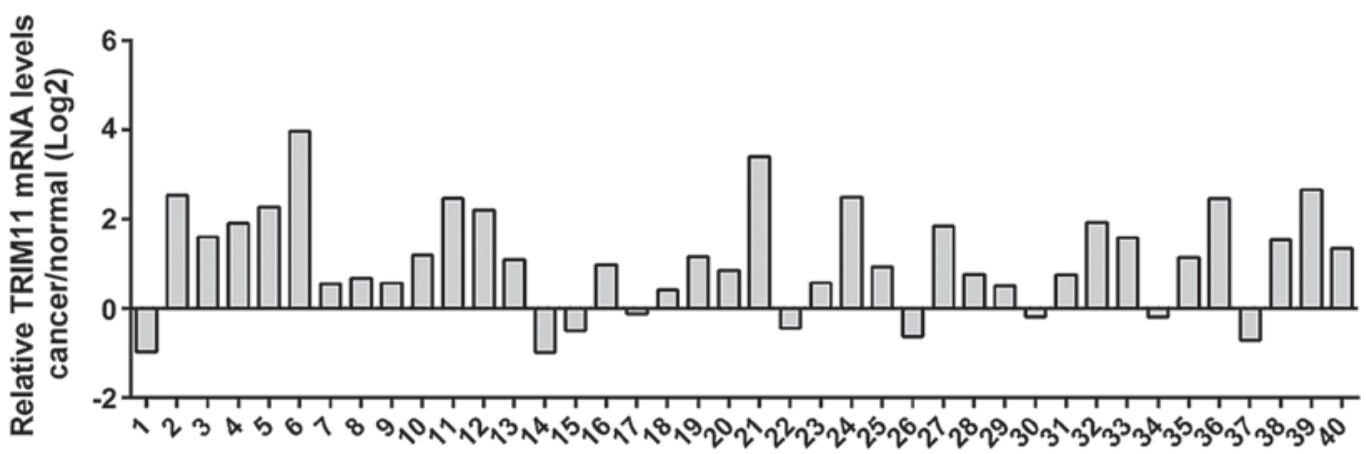

Patients

Figure 1. TRIM11 mRNA expression in ovarian cancer tissues. TRIM11 mRNA levels in ovarian cancer and matched normal tissues from 40 patients were determined by real-time PCR, with GAPDH as a control. The y-axis is $\log 2$ (tumor/normal) value. Positive $\log 2$ value indicated increased upregulation of TRIM11 in cancer tissues while negative $\log 2$ indicated downregulation of TRIM11 in cancer tissues. TRIM11, tripartite motif-containing 11.

A

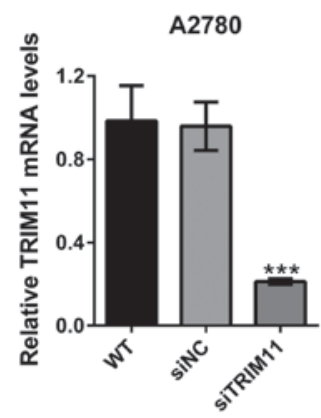

B
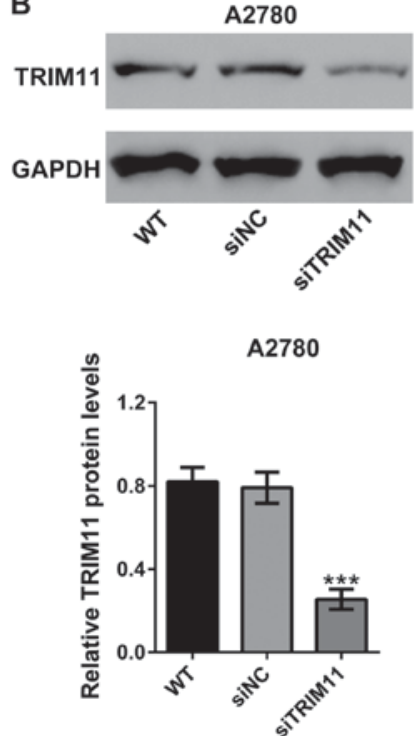

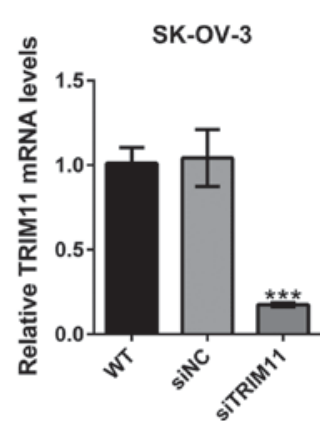

SK-OV-3

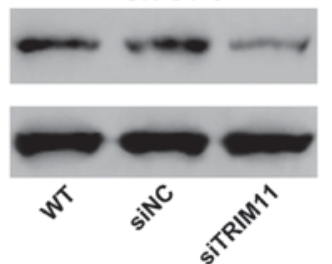

SK-OV-3

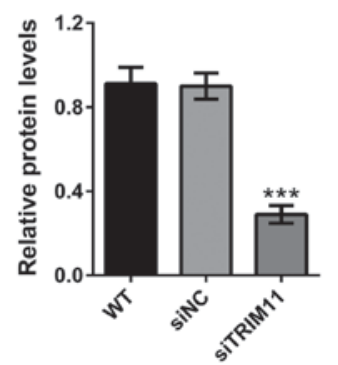

Figure 2. TRIM11 expression was repressed by specific siRNA transfection. A2780 and SK-OV-3 cells were transfected with siNC or TRIM11 siRNA (siTRIM11). (A) At $48 \mathrm{~h}$ after transfection, TRIM11 mRNA levels were determined by real-time PCR analysis. (B) TRIM11 protein levels were determined by western blot analysis with GAPDH as a loading control. Three independent experiments were performed for quantification (mean \pm standard deviation) (lower panel). ${ }^{* * *} \mathrm{P}<0.001$ vs. siNC. TRIM11, tripartite motif-containing 11; siRNA, small interfering RNA; siNC, negative control siRNA-transfected cells; siTRIM11, TRIM11 siRNA-transfected cells; WT, wild-type cells.

Downregulation of TRIM11 expression inhibits ovarian cancer cell proliferation. To explore whether TRIM11 plays

A A2780

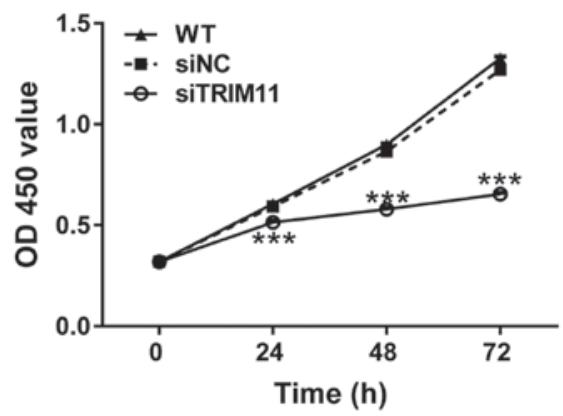

B

SK-OV-3

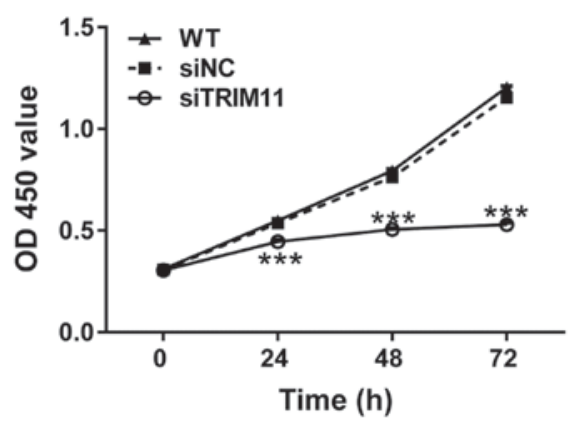

Figure 3. Knockdown of TRIM11 reduced ovarian cancer cell proliferation. (A) A2780 and (B) SK-OV-3 cells were transfected with siTRIM11 or siNC. Cell proliferation was detected by CCK-8 assay at 0,24, 48 and $72 \mathrm{~h}$ after transfection. ${ }^{* * *} \mathrm{P}<0.001$ vs. siNC. TRIM11, tripartite motif-containing 11 ; siTRIM11, TRIM11 siRNA-transfected cells; siNC, negative control small interfering RNA-transfected cells; CCK-8, Cell Counting Kit-8; WT, wild-type cells.

a role in the growth of ovarian cancer cells, CCK-8 assay was performed after siRNA transfection. The proliferation of A2780 and SK-OV-3 cells was remarkably repressed at 24, 48 and $72 \mathrm{~h}$ after siTRIM11 transfection (Fig. 3). These results indicated the inhibitory role of TRIM11-siRNA in the proliferation of ovarian cancer cells.

Knockdown of TRIM11 induces ovarian cancer cell apoptosis. Determining whether TRIM11 affects apoptosis of ovarian cancer cells, percentages of apoptotic cells were 
A

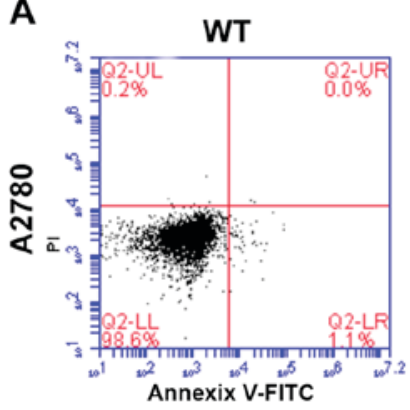

B

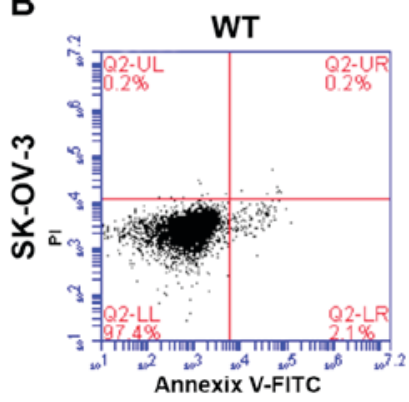

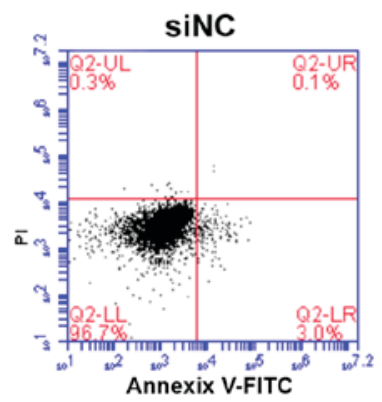
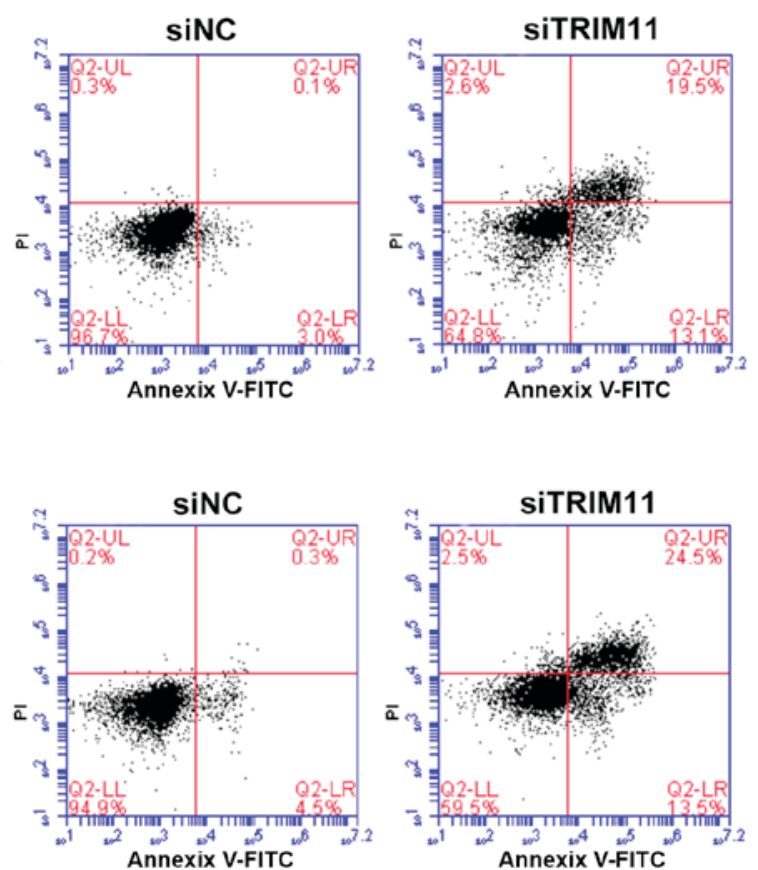
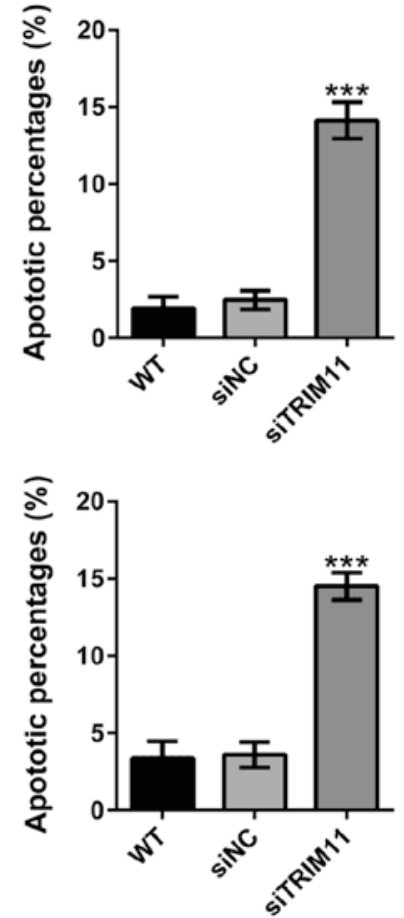

Figure 4. Suppressing TRIM11 expression induces ovarian cancer cell apoptosis. (A) A2780 and (B) SK-OV-3 cells were transfected with indicated siRNA. After 48-h of culture, cells were collected, stained with Annexin V/PI and analyzed by flow cytometry. Three independent experiments were performed for quantification (mean \pm standard deviation). ${ }^{* * *} \mathrm{P}<0.001$ vs. siNC. TRIM11, tripartite motif-containing 11; siRNA, small interfering RNA; PI, propidium iodide; siNC, negative control siRNA-transfected cells; WT, wild-type cells; siTRIM11, TRIM11 siRNA-transfected cells.

A
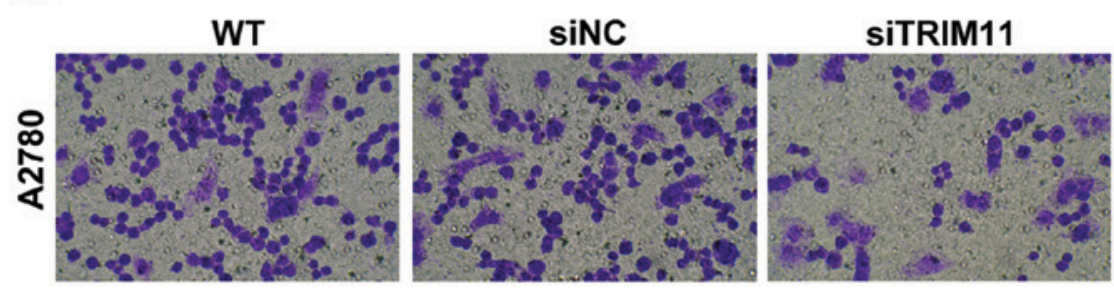

B
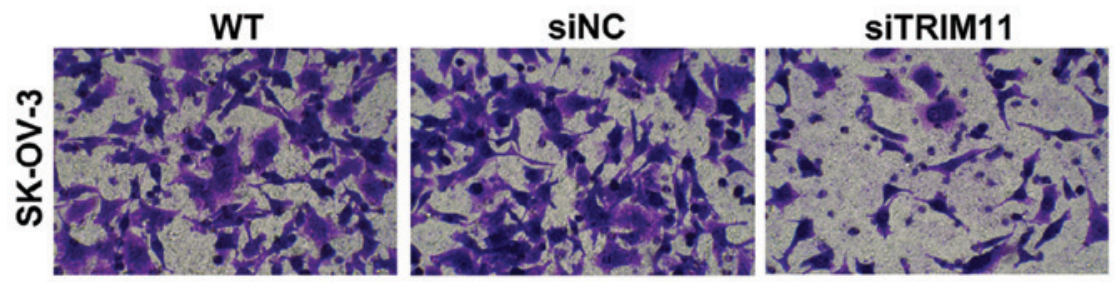
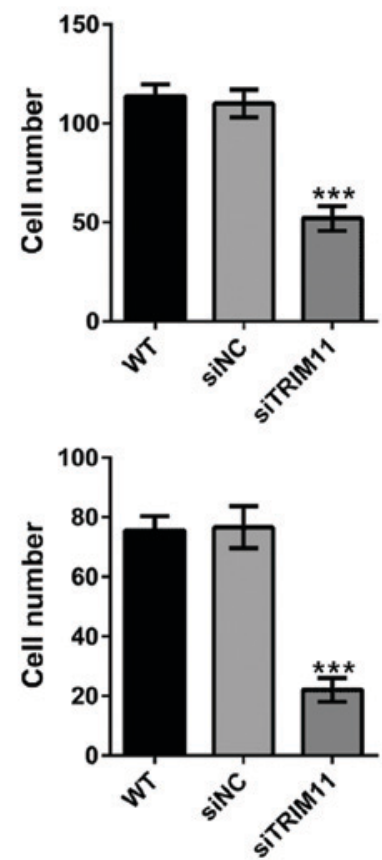

Figure 5. Knockdown of TRIM11 inhibits ovarian cancer cell invasion. Transwell invasion assay was performed in (A) A2780 and (B) SK-OV-3 cells. Three

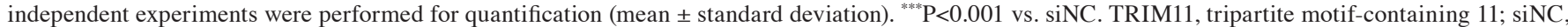
negative control small interfering RNA-transfected cells; WT, wild-type cells; siTRIM11, TRIM11 siRNA-transfected cells.

assessed in TRIM11 knockdown cells by Annexin V-FITC/PI staining assay. From flow cytometry analysis, it can be seen that compared with corresponding control cells (siNC), knockdown of TRIM11 in A2780 cells (Fig. 4A) markedly reduced cell apoptosis. Similar results were received in SK-OV-3 cells (Fig. 4B).
Depletion of TRIM11 inhibits ovarian cancer cell invasion. Using a Matrigel-coated Transwell, we evaluated the changes in ovarian cancer cell invasion after TRIM11 siRNA transfection. As shown in Fig. 5, depletion of TRIM11 in A2780 and SK-OV-3 cells led to markedly reduced cell apoptosis of 
A

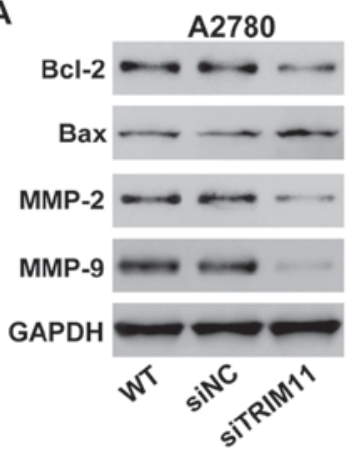

B

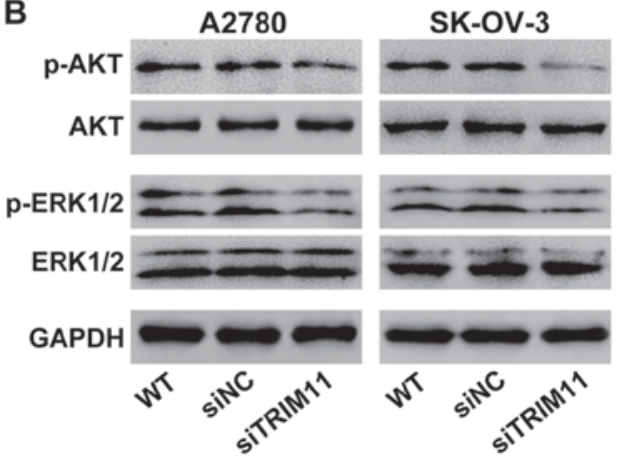

A2780

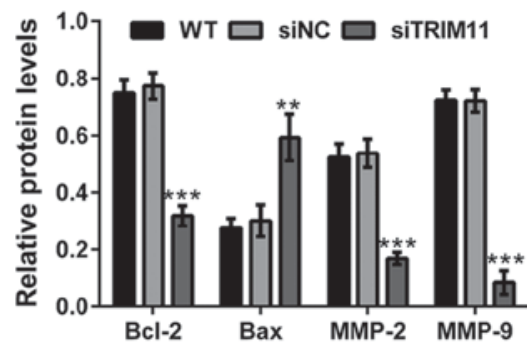

A2780

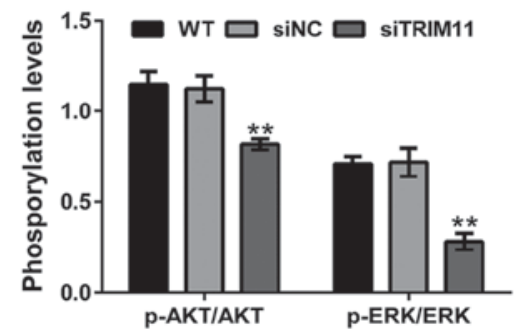

SK-OV-3

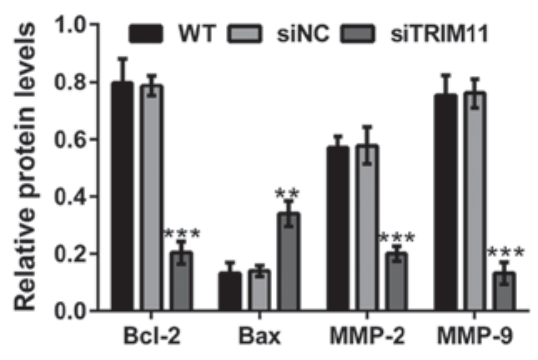

Figure 6. Effects of TRIM11 knockdown on the protein expression of (A) apoptosis and invasion-related regulators, and (B) the activation of AKT and ERK in ovarian cancer cells. Western blot analysis was performed with GAPDH as a loading control. Three independent experiments were performed for quantification (mean \pm standard deviation) (right panel). ${ }^{* *} \mathrm{P}<0.01$ and ${ }^{* * *} \mathrm{P}<0.001$ vs. siNC. TRIM11, tripartite motif-containing 11 ; WT, wild-type cells; siNC, negative control small interfering RNA-transfected cells; siTRIM11, TRIM11 siRNA-transfected cells.

invasion. These data revealed that TRIM11-siRNA play an inhibitory role on ovarian cancer cell invasion.

Effects of TRIM11 knockdown on the expression of apoptosis and invasion related proteins. The protein levels of cell apoptosis and invasion related proteins by western blot analysis (Fig. 6A) were evaluated. The levels of protein of Bcl-2, MMP-2 and MMP-9 were markedly suppressed in A2780 and SK-OV-3 cells with TRIM11 knockdown, but Bax was remarkably increased in TRIM11 knockdown cells.

Knockdown of TRIMI1 suppresses the activity of AKT and ERK in ovarian cancer cells. The effects of TRIM11 on AKT and ERK signaling have been studied in glioma cells (8) and lung cancer cells (9). Fig. 6B, shows that transfection of TRIM11 siRNA in ovarian cancer cells obviously suppressed the phosphorylation of AKT and ERK, but does not show any effects on the levels of AKT and ERK expression.

\section{Discussion}

Dysregulated expression of several members of TRIM proteins has been found in ovarian cancer tissues (11-14). The diagnostic and prognostic values of TRIM11 have been reported in gliomas (8), lung cancer (9) and colon cancer (10). Here, we reported that TRIM11 mRNA was efficient in ovarian cancer tissues (Fig. 1). Next, we studied the functions of TRIM11 in ovarian cancer by knockdown its expression with specific siRNA. Reduced expression of TRIM11 significantly suppressed proliferation of cells (Fig. 3) and invasion (Fig. 5), and induced apoptosis (Fig. 4). These data were consistent with previous investigation in other types of human cancer cells (8-10). The present study together with the previous findings suggested the important role of TRIM11 in cancer biology.

Members of Bcl-2 family are critical mediators for cell apoptosis promoting cell survival (e.g., Bcl-2 and Bcl-xL) and initiation of apoptosis (e.g., Bax and Bak) (15). Members of MMP family degrade extracellular matrix and cell surface proteins, thus playing an important role in cancer cell invasion (16). High levels of MMP-2 and MMP-9 predict poor survival in patients at advanced-stage of ovarian cancer (17). The activation of AKT and ERK pathways is involved in the regulation of Bcl-2 family $(18,19)$ and MMP family proteins $(20,21)$. Selective targeting of AKT or ERK pathways may block cancer progression (22-24). The effects of TRIM11 on AKT and ERK signaling have been studied in gliomas (8) and lung cancer cells (9). In the present study, we showed that knockdown of TRIM11 reduced phosphorylated levels of AKT and ERK, and the protein levels of Bcl-2, MMP-2 and MMP-9, whereas increased the protein levels of Bax in ovarian cancer cells (Fig. 6), suggesting that in ovarian cancer cells, targeting TRIM11 could be a successful anticancer strategy.

In conclusion, the expression of TRIM11 in ovarian cancer tissues was remarkably increased compared to normal adjacent tissues. The proliferation and invasion of ovarian cancer cells can be suppressed in ovarian cancer cells by depleting of TRIM11. We also illustrated that TRIM11 plays a key role and perform the functions by adjusting the levels of AKT and ERK. 


\section{References}

1. Siegel R, Naishadham D and Jemal A: Cancer statistics, 2013. CA Cancer J Clin 63: 11-30, 2013.

2. Heintz AP, Odicino F, Maisonneuve P, Quinn MA, Benedet JL, Creasman WT, Ngan HY, Pecorelli S and Beller U: Carcinoma of the ovary. FIGO 26th annual report on the results of treatment in gynecological cancer. Int J Gynaecol Obstet 95 (Suppl 1): 161-192, 2006.

3. Hatakeyama S: TRIM proteins and cancer. Nat Rev Cancer 11: 792-804, 2011

4. Niikura T, Hashimoto Y, Tajima H, Ishizaka M, Yamagishi Y, Kawasumi M, Nawa M, Terashita K, Aiso S and Nishimoto I: A tripartite motif protein TRIM11 binds and destabilizes Humanin, a neuroprotective peptide against Alzheimer's disease-relevant insults. Eur J Neurosci 17: 1150-1158, 2003.

5. Hong SJ, Chae H, Lardaro T, Hong S and Kim KS: Trim11 increases expression of dopamine beta-hydroxylase gene by interacting with Phox 2b. Biochem Biophys Res Commun 368: 650-655, 2008

6. Tuoc TC and Stoykova A: Trim11 modulates the function of neurogenic transcription factor Pax6 through ubiquitin-proteosome system. Genes Dev 22: 1972-1986, 2008.

7. Ishikawa H, Tachikawa H, Miura $\mathrm{Y}$ and Takahashi N: TRIM11 binds to and destabilizes a key component of the activator-mediated cofactor complex (ARC105) through the ubiquitin-proteasome system. FEBS Lett 580: 4784-4792, 2006.

8. Di K, Linskey ME and Bota DA: TRIM11 is overexpressed in high-grade gliomas and promotes proliferation, invasion, migration and glial tumor growth. Oncogene 32: 5038-5047, 2013.

9. Wang X, Shi W, Shi H, Lu S, Wang K, Sun C, He J, Jin W, Lv X, Zou H and Shu Y: TRIM11 overexpression promotes proliferation, migration and invasion of lung cancer cells. J Exp Clin Cancer Res 35: 100, 2016.

10. Yin Y, Zhong J, Li S-W, Li JZ, Zhou M, Chen Y, Sang Y and Liu L: TRIM11, a direct target of miR-24-3p, promotes cell proliferation and inhibits apoptosis in colon cancer. Oncotarget 7: 86755-86765, 2016.

11. Sakuma M, Akahira J, SuzukiT, Inoue S, Ito K, Moriya T, SasanoH, Okamura K and Yaegashi N: Expression of estrogen-responsive finger protein (Efp) is associated with advanced disease in human epithelial ovarian cancer. Gynecol Oncol 99: 664-670, 2005.
12. Ma Y, Wei Z, Bast RC Jr, Wang Z, Li Y, Gao M, Liu Y, Wang X, Guo C, Zhang L and Wang X: Downregulation of TRIM27 expression inhibits the proliferation of ovarian cancer cells in vitro and in vivo. Lab Invest 96: 37-48, 2016.

13. Santin AD, Zhan F, Bellone S, Palmieri M, Cane S, Bignotti E, Anfossi S, Gokden M, Dunn D, Roman JJ, et al: Gene expression profiles in primary ovarian serous papillary tumors and normal ovarian epithelium: identification of candidate molecular markers for ovarian cancer diagnosis and therapy. Int J Cancer 112: 14-25, 2004.

14. Tan H, Liu Z, Qi J and Chu G: Tripartite motif 16 inhibits the migration and invasion in ovarian cancer cells. Oncol Res 25: 551-558, 2017.

15. Reed JC: Regulation of apoptosis by bcl-2 family proteins and its role in cancer and chemoresistance. Curr Opin Oncol 7: 541-546, 1995.

16. Leber MF and Efferth T: Molecular principles of cancer invasion and metastasis (Review). Int J Oncol 34: 881-895, 2009.

17. Davidson B, Goldberg I, Gotlieb WH, Kopolovic J, Ben-Baruch G, Nesland JM, Berner A, Bryne M and Reich R: High levels of MMP-2, MMP-9, MT1-MMP and TIMP-2 mRNA correlate with poor survival in ovarian carcinoma. Clin Exp Metastasis 17: 799-808, 1999.

18. Downward J: PI 3-kinase, Akt and cell survival. Semin Cell Dev Biol 15: 177-182, 2004.

19. Boucher MJ, Morisset J, Vachon PH, Reed JC, Lainé J and Rivard N: MEK/ERK signaling pathway regulates the expression of $\mathrm{Bcl}-2, \mathrm{Bcl}-\mathrm{X}(\mathrm{L})$, and $\mathrm{Mcl}-1$ and promotes survival of human pancreatic cancer cells. J Cell Biochem 79: 355-369, 2000.

20. Jung C-H, Kim EM, Park JK, Hwang SG, Moon SK, Kim WJ and Um HD: Bmall suppresses cancer cell invasion by blocking the phosphoinositide 3-kinase-Akt-MMP-2 signaling pathway. Oncol Rep 29: 2109-2113, 2013.

21. Adya R, Tan BK, Punn A, Chen J and Randeva HS: Visfatin induces human endothelial VEGF and MMP-2/9 production via MAPK and PI3K/Akt signalling pathways: novel insights into visfatin-induced angiogenesis. Cardiovasc Res 78: 356-365, 2008.

22. Morgensztern D and McLeod HL: PI3K/Akt/mTOR pathway as a target for cancer therapy. Anticancer Drugs 16: 797-803, 2005.

23. Kohno $\mathrm{M}$ and Pouyssegur J: Targeting the ERK signaling pathway in cancer therapy. Ann Med 38: 200-211, 2006.

24. Hill MM and Hemmings BA: Inhibition of protein kinase B/Akt. implications for cancer therapy. Pharmacol Ther 93: 243-251, 2002. 